\title{
Imaging while reading text: A study of individual differences
}

\author{
MICHEL DENIS \\ Université de Paris VIII, 93526 Saint-Denis Cedex 2, France
}

\begin{abstract}
In a series of four experiments, reading time of texts and memory for their informational content were studied in relation to readers' imagery abilities. The main prediction was that if high imagers (HIs) tend to elaborate images expressing the semantic content of the text while reading, then they should require more time to read imageable material than low imagers (LIs). And in fact, when subjects read a descriptive/narrative text, HIs not only read more slowly, but remembered the text better than LIs. In contrast, when subjects read abstract, nonimageable material, there was no difference between the two groups in reading times or memory. Additional experiments provided further support for the claim that HIs spent more time elaborating images while they read descriptive/narrative text.
\end{abstract}

Many readers claim that their reading of descriptive or narrative texts is accompanied by a sequence of visual images that express the semantic content of the text. Such images influence later memory, as has been demonstrated repeatedly in the past years (e.g., Denis, 1979; Paivio, 1971). Both recall and recognition of information in texts are highly dependent on experimental variations of imagery activity. On the whole, recall of a text increases when subjects produce more images while reading (Anderson \& Kulhavy, 1972). Furthermore, subjects classified as "high imagers" (HIs) are able to recall or recognize more items of information than are "low imagers" (LIs), and performance of LIs significantly increases when they are instructed to form visual images during reading (Chaguiboff \& Denis, 1981; Kulhavy \& Swenson, 1975; Lesgold, McCormick, \& Golinkoff, 1975; Levin \& Divine-Hawkins, 1974; Pressley, 1976).

Given the finding that imagery variables affect memory for written material, one is led to wonder about the role of imagery in reading. Theories of reading commonly stress the constructive character of processes that occur during text reading. Readers are said to "construct meaning" by discovering the relations between the concepts referred to by different parts of the text. Readers are said to "construct images" when they are processing descriptive/narrative texts. What is the

This research was conducted in the Laboratoire de Psychologie, E.R.A. au C.N.R.S. No. 235, Université de Paris VIII, as a contribution to the A.T.P. No. 3580 (Travail de l'Eleve). Danielle Le Taillanter efficiently contributed to collection and analysis of the data. This paper was written while the author was a Fulbright Visitor at the Department of Psychology, Brandeis University, Waltham, Massachusetts. The author wishes to thank Steve Kossiyn for his helpful comments on this paper and his patience in revising it. Connie Greenbaum deserves further thanks for her suggestions on the final manuscript. Requests for reprints should be sent to Michel Denis, Laboratoire de Psychologie, E.R.A. au C.N.R.S. No. 235, Universite de Paris VIII, 2, rue de la Liberté, 93526 Saint-Denis Cedex 2, France. relationship between these two kinds of constructive activity? Is imagery an inherent part of meaning? Or is it partially autonomous with respect to meaning? Is image elaboration an obligatory process from which meaning is constructed? Or is meaning foremost, and imagery just a circumstantial, optional expression of meaning?

None of these questions seems to receive definite answers from current theories or from available data. The hypothesis that imagery is an inherent component of meaning (cf. Mowrer, 1977; Paivio, 1971) has been criticized by theorists stressing the abstract, amodal nature of meaning and arguing for propositions as the most appropriate format of representation for meaning (cf. Anderson \& Bower, 1973; Pylyshyn, 1973). However, the data indicate that many people represent at least a part of the semantic information garnered while reading prose as images. Thus, a full understanding of reading entails understanding the process by which imagery is evoked during the course of reading. This process may well affect not just later memory, but actual comprehension of the material as it is being read.

One property of images that may affect comprehension is their duration. Very little attention has been paid to the duration of mental images, perhaps because it has not seemed relevant for the processes studied to date. Like any psychological process, imagery activity has a time course. Although the time to form images of named stimuli has now been widely investigated, the duration of the image once it has been formed has received only scant attention to date (see Denis, 1982). It is of interest, however, that the imageability of words has opposite effects on the time needed to form images and the time they can be maintained: Images can be formed in response to words rated high on imagery value very quickly, and those images are maintained for relatively long times, whereas more time is required to form images in response to low-imagery words, and those images "vanish" more rapidly (Simpson \& Bergin, Note 1). 
On the basis of these considerations, we developed two hypotheses: First, we considered the temporal characteristics of imagery in reading texts. We hypothesized that a reader who is reading a text with a descriptive, concrete content will develop a series of successive images that depict the scenes and episodes described in the text. When those descriptions denote, for example, physical data (descriptions of characters, of landscapes, etc.) or narrative data (descriptions of actions, of events, etc.), the reader will tend to develop such a series of images. Thus, even if we consider that all or a part of the imagery activities develop in parallel with semantic processing of text, we may hypothesize that texts containing such concrete descriptions and episodes will evoke a larger "amount" of imagery activity, and hence will require more time to read, than texts devoid of any concrete descriptions and episodes, assuming an equivalent level of text "difficulty."

Our second hypothesis concerns individual differences in imaging verbal material. In this domain, as in others, human abilities are distributed along a continuum. At one extreme, some people are presumably strongly oriented toward imagery activities. These people are prone to transform most verbal information into rich, vivid visual representations, and they tend to devote a great deal of their cognitive resources to the elaboration and inspection of their own images, and so on. At the other extreme, some people may never elaborate images and may have no spontaneous tendency to use visual images in cognitive activities. The question is: What shall we expect from subjects classified as HIs when they are reading a descriptive/narrative text in natural or close-to-natural reading conditions? Our hypothesis is that if total reading time includes the time to form visual images, then these activities will tend to occupy a longer time in HIs than in LIs. This prediction rests on the notion that HIs will be able to maintain more vivid, detailed images that will consume more time than is spent by LIs. If we assume that the other abilities involved in reading are equally distributed in both groups (perceptual abilities, visual decoding of letter strings, abilities in semantic analysis, previous knowledge, etc.), then the longer time devoted to imagery activity should be reflected in longer total reading times for HIs.

We have reason to expect this result from the findings of Chaguiboff and Denis (1981). In that experiment, adult subjects read a 2,000-word narrative telling the story of an automobile trip, with numerous episodes describing various characters, scenes, and events. Reading speed was self-paced. After reading the text, the subjects had to recognize words or pictures describing items of information mentioned in the text. Furthermore, the subjects had to complete Marks' (1973) Vividness of Visual Imagery Questionnaire (VVIQ), which allowed Chaguiboff and Denis to distinguish HIs from LIs. Although the main thrust of the experiment was to observe the effects of imagery ability on further recognition, spontaneous reading times of subjects also were recorded. HIs had longer reading times than LIs, with a difference of about $+10 \%$. In fact, imagery ability (as reflected by VVIQ scores) and reading time were positively correlated.

The present experiments were designed in part to examine the generality of the previously observed phenomenon, using new experimental material. In addition, we attempted to implicate imagery in this process in a more direct way.

\section{EXPERIMENT 1}

The main purpose of this experiment was to replicate our earlier finding that people classified as HIs take more time to read a descriptive/narrative text when they are free to read at their own speed. In this experiment, memory for informational content was also tested. Increased use of imagery during reading should result in increasingly better memory (see Kulhavy \& Swenson, 1975; Levin \& Divine-Hawkins, 1974); thus, this measure will provide converging evidence that imagery was used.

\section{Method}

Materials. An approximately 2,200-word narrative in French was freely adapted from Farmer Palmer's Wagon Ride (Steig, 1974). It told the story of a farmer who rides to a village to sell his crops and shop, and who meets with a series of incidents traveling home. Characters were described with many concrete details, and events were related in a descriptive manner. This kind of a text seemed especially likely to elicit visual images during reading.

Procedure. The subjects were tested individually. They first were asked to read the text typewritten on a five-page booklet. With the aim of approximating spontaneous reading as nearly as possible, the subjects were instructed to read the text carefully, at their own pace, without rereading. They were told that they would have to answer questions at the end of the experiment. The experimenter sat at the opposite side of the room and, without the subjects' knowledge, recorded the time between subjects' opening the booklet and their turning over the last page. The experimenter also watched that the subjects did not engage in some interfering activity likely to affect reading time. For instance, some subjects unexpectedly lit cigarettes and thus had to be discarded and replaced. Immediately after reading, the subjects completed a 24 -item two-alternative forced-choice test on characters and events discussed in the text. Each item referred to a fact that had been explicitly stated in the text (e.g., "In Antoine's wagon there were: (a) carrots; (b) turnips."). The subjects were told that in every case one of the two answers was right, and they were requested not to respond by chance but to check an "I don't know" answer in case of indecision. The 24 questions were presented in the same order as was used to present the corresponding information in the text. After having completed the test, the subjects completed the French adaptation of Marks' (1973) VVIQ.

Subjects. The subjects were $\mathbf{4 2}$ introductory students in psychology ( 13 male). All were native speakers of French, as were all the subjects in the following experiments of this series.

\section{Results and Discussion}

Subjects were initially classified as HIs or LIs according to whether their VVIQ scores were above or below the group's median. The HIs' spontaneous reading times were longer than the LIs' times, $538 \mathrm{sec}$ vs. $472 \mathrm{sec}$, respectively; this $+14 \%$ difference between the two groups was significant $[\mathrm{t}(40)=2.15, \mathrm{p}<.05]$. The 
relationship between imagery abilities and reading times was also evident in the significant positive correlation between VVIQ scores and reading times $[\mathrm{r}(41)=.37$, $\mathrm{p}<.02]$.

For the purpose of further analyses (described below), reading times of the 10 highest VVIQ scorers were compared with those of the 10 lowest VVIQ scorers. Mean reading times were $575 \mathrm{sec}$ for the HIs vs. $463 \mathrm{sec}$ for the LIs; this $+24 \%$ difference between the two groups was significant $[t(18)=2.49, \mathrm{p}<.05]$.

These results support the hypothesis that individuals prone to using imagery have longer reading times for descriptive/narrative texts. The extra time devoted to reading presumably reflects, at least partly, the extra imagery activity engaged in by HIs when they read highly imageable material.

We must also take into account the fact that HIs are individuals who are able to elaborate images with shorter latencies than LIs when they are required to image to verbal stimuli (see Ernest \& Paivio, 1971; Rehm, 1973). This aspect of their abilities should lead, in principle, to shorter reading times. However, these subjects are also presumably able to maintain their images longer after they have constructed them. In fact, if one considers the results of experiments comparing generation latencies and durations of images to single high-imagery words, the absolute values of generation latencies are much shorter than those of durations, in a proportion of 1 to $1.7-5.9$ (see Simpson \& Bergin, Note 1). Thus, a possible temporal "saving" gained on the time HIs require to generate images should be largely counterbalanced by the extra time devoted to maintenance of images.

In addition to taking longer to read, HIs also obtained higher scores on the test than did LIs [14.7 vs. 11.8, $t(40)=2.03, p<.05$, in terms of the entire population; 15.9 vs. $11.7, \mathrm{t}(18)=2.14, \mathrm{p}<.05$, in terms of the 10 highest HIs and the 10 lowest LIs]. These results replicate the well known phenomenon of higher memory performances in subjects prone to constructing images during information encoding (see Ernest, 1977). Thus, this finding provides support for the inference that HIs did in fact spontaneously engage in more imagery activity during reading than did LIs.

\section{EXPERIMENT 2}

Although the results thus far are consistent with the hypothesis that some people evoke more imagery during reading than others, this is not the only interpretation. HIs, for example, could be slower readers in general. If this is the case, then HIs should systematically tend to devote longer times than LIs to reading any text, and a difference in reading times should still be observed even for texts unlikely to elicit any images. Alternatively, if imagery use was at the root of the differences in reading times, there should be no time difference between HIs and LIs for texts that do not lend themselves to conversion into visual images. Thus,
Experiment 1 was repeated, but subjects now read an abstract text instead of the previous one.

\section{Method}

Materials. The text used in Experiment 2 was on objective vs. subjective approaches in psychology. It was taken from the Manuel de Psychologie (Guillaume, 1963). All illustrative examples and comments were discarded, as well as titles and headings, so that the final version consisted only of abstract sentences that were unlikely to elicit any visual images. A typical excerpt is the following: "In the issues which are studied in psychology, one has the aim, as in natural sciences, to describe facts and to determine their conditions, that is other facts whose observation points to their steady relationship with the former ones; in other words, one has the aim to set up laws." Taking into account the aridity of the text, we shortened it to 1,600 words, which was slightly shorter than the text used in Experiment 1. This difference did not seem critical, given that we did not plan to compare the absolute reading times for both texts, but only to examine reading time differences for the two groups of subjects for each text separately.

Procedure. A new group of subjects was asked to read the abstract text in experimental conditions strictly identical with those of Experiment 1. After self-paced reading, which was recorded without the subjects' knowledge, the subjects took a 24-item two-alternative forced-choice test. Each question was a repetition of a sentence of the text, with a missing word, and the subjects had to check which of two words had originally appeared in the text (e.g., "The pure objective method approaches psychological facts in their: (a) function; (b) nature."). The distractor term was never a synonym of the correct answer. The subjects were requested not to respond by chance but to check an "I don't know" answer in case of indecision. After completing the text, the subjects completed the French adaptation of Marks' (1973) VVIQ.

Subjects. The subjects were 42 introductory students in psychology (10 male), who had not participated in the previous experiment.

\section{Results and Discussion}

The median value of the VVIQ scores (44.10) was virtually identical to the corresponding value in Experiment 1 (44.50). Furthermore, similar dispersion patterns attested to the similarity of both experimental populations. Thus, the populations were comparable.

There was virtually no difference between HIs and LIs in their spontaneous reading times, respectively, $488 \mathrm{sec}$ and $484 \mathrm{sec}[\mathrm{t}(40)<1]$, in terms of the entire population. The correlation between imagery ability and reading time was effectively zero $[\mathrm{r}(41)=.04]$. In terms of the 10 highest HIs and the 10 lowest LIs, reading times were, respectively, $458 \mathrm{sec}$ and $480 \mathrm{sec}$ $[\mathrm{t}(18)<1]$.

In order to test the interaction between imagery ability and type of text (narrative vs. abstract), the data from Experiments 1 and 2 were combined in a single analysis of variance. It was for the purposes of conducting such cross-experiment comparisons that the two subgroups, that is, the top 10 vs. the bottom 10 VVIQ scorers, were contrasted. This was done to avoid the ambiguity of median splits, since some of the subjects on different sides of the split may have had very similar imagery scores. Furthermore, subjects classified as LIs in one experiment could be classified as HIs in another experiment. Rather than pooling all subjects from a 
pair of experiments and doing a median split on the VVIQ scores of the larger population, this resulting in analysis difficulties, such as unequal numbers of subjects in different conditions, it seemed preferable to rely on comparisons between the 10 highest HIs and the 10 lowest LIs in each experiment.

According to the analysis of the combined data from Experiments 1 and 2, the interaction between imagery ability and type of text proved to be significant $[\mathrm{F}(1,36)=5.92, \mathrm{p}<.025]$. The effects of imagery ability on reading times for the narrative text were confirmed [partial comparison, $F(1,36)=8.33, \mathrm{p}<.01$ ] , as well as the lack of effect in the case of the abstract text $[F(1,36)<1]$. Thus, it is reasonable to conclude that the difference in reading times for the narrative text found in Experiment 1 genuinely reflects a difference in the time devoted to imagery activity.

Considering the number of correct responses to the test, there again was no difference between HIs and LIs [respectively, 13.7 vs. $14.0, \mathrm{t}(40)<1$, in terms of the entire population; 14.1 vs. $13.8, \mathrm{t}(18)<1$, in terms of the 10 highest HIs and the 10 lowest LIs]. Indeed, there is no reason why individual differences in imagery ability should be reflected in memory for information that was encoded without using imagery. On the whole, individual differences in imagery ability seem to affect processing of verbal material only insofar as this material is likely to elicit imagery activity.

\section{EXPERIMENT 3}

We have claimed that the difference in reading times between HIs and LIs observed in Experiment 1 was, in fact, due to HIs' maintaining images for longer durations. If so, then by eliminating this component of the process, HIs should speed up considerably. Furthermore, their improvement should be more dramatic than LIs, who held images for briefer durations initially. Thus, HIs, who are slower than LIs in a self-paced reading situation, should speed up much more than LIs when asked to read quickly a text likely to elicit visual images. Further, if the earlier difference was due to imagery processes, when such processes are minimized there should be no difference in reading times between the two groups. In Experiment 3, we measured reading times for the text used in Experiment 1 in HIs and LIs when they were instructed to read the text as fast as possible.

\section{Method}

Materials. The text was the same as the text used in Experiment 1 .

Procedure. A new group of subjects was asked to read the text in experimental conditions identical with those of Experiment 1 , with the only modification being that they were instructed to read as fast as possible. These instructions were repeated a second time as a paraphrase. After reading, the subjects completed the test on characters and events, and then they completed the VVIQ.

Subjects. The subjects were 42 introductory students in psychology (11 male), who had not participated in either of the previous experiments.

\section{Results and Discussion}

The median value of the VVIQ scores (44.25) and dispersion patterns were very similar to those of groups used in Experiments 1 and 2.

Reading times of the 10 highest HIs were compared with those of the 10 lowest LIs. The results were straightforward: HIs read at a speed not significantly different from LIs $[t(18)=1.01, p>.10]$. The results are presented in Table 1, which also presents the reading times from Experiment 1 for purposes of comparison.

Data from both experiments were considered in a single analysis of variance. Not surprisingly, subjects who were told to read quickly in fact read more quickly $[F(1,36)=5.17, p<.05]$. Of more interest is the fact that the interaction between groups and experiments was significant $[F(1,36)=6.22, p<.025]$. There was no difference in time for LIs in the two experiments [partial comparison, $\mathrm{F}(1,36)<1$ ], whereas HIs were $26 \%$ faster than the HIs in Experiment $1[\mathrm{~F}(1,36)=$ $11.36, p<.005]$. No other effects were significant.

The preceding results do not allow us to infer precisely when HIs save time in fast-reading conditions. One may assume that if they still construct images, they tend to shorten the length of time that they maintain them; in addition, they may decrease the time spent constructing the images initially. However, because the absolute time to generate images is much shorter than

Table 1

Mean Reading Times (in Seconds) and Recognition Scores

\begin{tabular}{|c|c|c|c|c|c|c|c|}
\hline \multirow[b]{2}{*}{ Experiment } & \multirow[b]{2}{*}{ Text } & \multirow[b]{2}{*}{ Instructions } & & \multirow{2}{*}{$\begin{array}{c}\text { Reading } \\
\text { Times }\end{array}$} & \multicolumn{3}{|c|}{ Recognition Scores } \\
\hline & & & & & Correct & Incorrect & Don't Know \\
\hline 1 & Narrative & Self-Paced & $\begin{array}{l}\text { LIs } \\
\text { HIs }\end{array}$ & $\begin{array}{l}463 \\
575\end{array}$ & $\begin{array}{l}11.7 \\
15.9\end{array}$ & $\begin{array}{l}3.8 \\
3.4\end{array}$ & $\begin{array}{l}8.5 \\
4.7\end{array}$ \\
\hline 2 & Abstract & Self-Paced & $\begin{array}{l}\text { LIs } \\
\text { HIs }\end{array}$ & $\begin{array}{l}480 \\
458\end{array}$ & $\begin{array}{l}13.8 \\
14.1\end{array}$ & $\begin{array}{l}5.9 \\
7.4\end{array}$ & $\begin{array}{l}4.3 \\
2.5\end{array}$ \\
\hline 3 & Narrative & Fast Reading & $\begin{array}{l}\text { LIs } \\
\text { HIs }\end{array}$ & $\begin{array}{l}470 \\
426\end{array}$ & $\begin{array}{l}10.3 \\
13.9\end{array}$ & $\begin{array}{l}3.8 \\
5.7\end{array}$ & $\begin{array}{l}9.9 \\
4.4\end{array}$ \\
\hline 4 & Narrative & Imagery & $\begin{array}{l}\text { LIs } \\
\text { HIs }\end{array}$ & $\begin{array}{l}649 \\
685\end{array}$ & $\begin{array}{l}16.5 \\
16.8\end{array}$ & $\begin{array}{l}3.4 \\
4.7\end{array}$ & $\begin{array}{l}4.1 \\
2.5\end{array}$ \\
\hline
\end{tabular}


the time spent maintaining them, as was previously mentioned, it is likely that decreased time in holding images is the main factor responsible for decreasing the total reading time in HIs in Experiment 3.

Perhaps the most interesting finding here emerged from the analysis of the test scores (see Table 1). HIs obtained higher scores than LIs, respectively, 13.9 vs. $10.3[\mathrm{t}(18)=2.73, \mathrm{p}<.02]$, as was previously the case in Experiment 1. This was true even though HIs required no more time than LIs to read the text. Further, when the data from Experiments 1 and 3 were considered in a single analysis of variance, the scores obtained did not differ from those found in Experiment $1[F(1,36)=$ $1.54, \mathrm{p}>.10]$. In fact, only the effects of imagery ability were significant $[F(1,36)=8.13, p<.01]$. The superiority of HIs was the same in the two experiments $[F(1,36)<1$ for the interaction between imagery abilities and instructions]. Thus, a sizable part of the imagery activity in HIs would not seem to be oriented toward mnemonic encoding of information. Further, the earlier findings on memory differences between HIs and LIs are not simply a consequence of HIs' spending more time encoding the material while reading.

\section{EXPERIMENT 4}

In the last experiment of this series, subjects were asked to read the text while constructing visual images of characters and events for every sentence. Such instructions should increase the total reading time for all subjects, but more so for LIs; HIs will use imagery spontaneously and, hence, should not perform much differently when told to do so. HIs might also have longer reading times than LIs, if only because their more vivid imagery would allow them to hold images longer.

\section{Method}

Materials. The text was the same as the text used in Experiments 1 and 3.

Procedure. A new group of subjects was asked to read the text. The instructions were the same as those used in Experiment 1, except that now the subjects were asked to construct visual images as rich and vivid as possible for places, characters, and actions presented in the text. With the exception of these instructions, which were repeated as a paraphrase, the experimental conditions were identical to those of Experiment 1. After reading, the subjects completed the test on characters and events and then completed the VVIQ

Subjects. The subjects were 42 introductory students in psychology (11 male), who had not participated in any of the previous experiments.

\section{Results and Discussion}

Although the median value of the VVIQ scores (47.00) was slightly higher than values previously computed, there were no significant differences among the measures taken in each experiment $[\mathrm{F}(3,164)=$ $1.49, \mathrm{p}>.10]$. Furthermore, none of the comparisons between the median in Experiment 4 and medians in Experiments 1 through 3 reached significance according to the median test $\left[\chi^{2}(1)=2.48,1.25\right.$, and 2.19 , respectively; $p>.10$ for all comparisons]. ${ }^{1}$
The reading times for the 10 highest HIs and the 10 lowest LIs are presented in Table 1. It is obvious that imagery instructions slowed down both HIs and LIs $[F(1,36)=10.66, p<.005]$. Although the interaction between imagery abilities and instructions was not significant $[\mathrm{F}(1,36)<1]$, planned comparisons testing the predicted pattern were performed: Whereas the HIs were only $19 \%$ slower than those in Experiment 1 $[F(1,36)=2.97, p>.05]$, the LIs were $40 \%$ slower $[F(1,36)=8.38, p<.01]$. There was, finally, no significant difference in overall reading times beween HIs and LIs in this experiment $[\mathrm{F}(1,36)<1]$.

Furthermore, imagery instructions improved the recognition scores $[F(1,36)=5.16, p<.05]$ between Experiments 1 and 4 . This effect, however, was essentially due to LIs, whose profit from imagery instructions was obvious $[F(1,36)=7.31, p<.01]$, whereas HIs' scores did not significantly differ in Experiments 1 and 4 $[F(1,36)<1]$. Finally, HIs and LIs in Experiment 4 did not differ in recognition scores, 16.8 and 16.5 , respectively $[F(1,36)<1]$.

\section{GENERAL DISCUSSION}

In conditions close to spontaneous reading conditions for a text likely to elicit images, individual differences in imagery abilities influence the temporal course of linguistic information processing. Subjects characterized as HIs seem to be inclined to use these abilities when they read a descriptive/narrative text, and this imagery activity requires extra time.

Several interpretations of this effect remain possible. An interpretation already mentioned in this paper is that although all subjects construct images equally frequently, HIs tend to maintain their images longer than LIs do. An alternate and compatible interpretation is that HIs' images are more complex, or richer in details, thus requiring further elaboration, which would be reflected by extra time. A third possibility is that LIs simply do not construct images as often as HIs, in contrast with the first interpretation. Further research is needed in order to discriminate among these different explanations.

Individual differences in imagery abilities also have significant effects on memory for information extracted from the text, with HIs having higher recognition scores. However, the relationship between imagery abilities and memory is not a simple one, since HIs are able to eliminate a sizable part of their imagery activity without significant impairment of recognition. So imagery activity developed by HIs in spontaneous reading conditions can be reduced without severely affecting memorization. This result leads us to wonder about the role of spontaneous imagery in mnemonic encoding. It appears, in fact, that at least a part of imagery activity developed during prose reading is not definitely directed toward memorization. This makes sense, given that in daily psychological life there are imagery activities 
whose function is not easy to characterize, unless we relate it with a kind of homeostasis of the mind, as is the case for daydreaming. These activities are distinct from imagery activities with definite cognitive purposes (e.g., imagery that accompanies perceptual anticipation, search in memory for figural information, spatial problem solving, etc.) It is a matter of fact that a great deal of the images elicited by narratives (novels, etc.) are not "goal directed." We certainly have to bear in mind this "hedonistic" aspect of imagery in many natural situations.

However, it is clear that imagery is not simply a "fifth wheel" spinning freely. Imagery remains a prime instrument for encoding figural information and thus must be considered as one of the cognitive strategies whose purpose is to maximize the probability of information encoding and storage. This role is evident in Experiment 4, in which individuals not inclined to produce images spontaneously are able to develop an imagery activity when instructed to do so (which is clear from lengthening of reading times) and thereby achieve recognition scores as high as the HIs' recognition scores.

\section{REFERENCE NOTE}

1. Simpson, H. M., \& Bergin, J. C. Imagery latency and duration. Paper presented at the meetings of the Canadian Psychological Association, St. John's, Newfoundland, June 1971.

\section{REFERENCES}

Anderson, J. R., \& Bower, G. H. Human associative memory. Washington, D.C: Winston, 1973.

Anderson, R. C., \& Kulhavy, R. W. Imagery and prose learning. Journal of Educational Psychology, 1972, 63, 242-243.

Chaguiboff, J., \& Denis, M. Activité d'imagerie et reconnaissance de noms provenant d'un texte narratif. L'Année Psychologique, 1981, 81, 69-86.

Denis, M. Les images mentales. Paris: Presses Universitaires de France, 1979.

Denrs, M. Les aspects temporels de l'activite d'imagerie. Psy. chologie Française, 1982, 27, 134-145.

ERNEST, C. H. Imagery ability and cognition: A critical review. Journal of Mental Imagery, 1977, 1, 181-216.

Ernest, C. H., \& Paivio, A. Imagery and verbal associative latencies as a function of imagery ability. Canadian Journal of Psychology, 1971, 25, 83-90.

Guillaume, P. Manuel de psychologie. Paris: Presses Universitaires de France, 1963.

Kulhavy, R. W., \& Swenson, I. Imagery instructions and the comprehension of text. British Journal of Educational Psychology, 1975, 45, 47-51.

Lesgold, A. M., McCormick, C., \& Golinkoff, R. M. Imagery training and children's prose learning. Journal of Educational Psychology, 1975, 67, 663-667.

Levin, J. R., \& Divine-Hawkins, P. Visual imagery as a proselearning process. Journal of Reading Behavior, 1974, 6, 23-30.

MARks, D. F. Visual imagery differences in the recall of pictures. British Journal of Psychology, 1973, 64, 17-24.

Mowrer, O. H. Mental imagery: An indispensable psychological concept. Journal of Mental Imagery, 1977, 1, 303-325.

PAIvio, A. Imagery and verbal processes. New York: Holt, Rinehart, \& Winston, 1971.

Pressley, G. M. Mental imagery helps eight-year-olds remember what they read. Journal of Educational Psychology, 1976, 68, 355-359.

Pylyshyn, Z. W. What the mind's eye tells the mind's brain: A critique of mental imagery. Psychological Bulletin, 1973, $80,1-24$.

REHM, L. P. Relationships among measures of visual imagery. Behavior Research and Therapy, 1973, 11, 265-270.

STEIG, W. Falmer Palmer's wagon ride. New York: Farrar, Straus, \& Giroux, 1974.

NOTE

1. The slight, recurrent tendency for VVIQ scores to be on the whole higher in Experiment 4 than in Experiments 1 through 3 may be an indication that the imagery that develops during reading as a result of following the instructions (Experiment 4) at least partially affects later rating on the VVIQ. Indeed, a kind of a "warm-up" effect from the previous imagery activity may slightly increase the amount of imagery developed in later tasks. However, this effect, if at all present, is consistently below significance. One way to alleviate such an effect would be to have subjects complete the VVIQ prior to reading the texts. However, this sequence would probably result in similar contamination effects from the imagery solicited during VVIQ rating on the imagery developed during reading texts. This effect would be much more problematic because it would interfere with the amount of imagery produced during reading, therefore interfering with the dependent variable used as an indirect measure of this imagery activity.

(Received for publication February 22, 1982; revision accepted July 29,1982 .) 